\title{
Pseudo ternary phase diagrams: a practical approach for the area and centroid calculation of stable microemulsion regions
}

\author{
Murat Sami Berkman' (D), Kadri Güleç² (i) \\ 'Anadolu University, Faculty of Pharmacy, Department of Pharmaceutical Technology, Eskișehir, Turkey \\ ${ }^{2}$ Anadolu University, Faculty of Pharmacy, Department of Analytical Chemistry, Eskișehir, Turkey
}

ORCID IDs of the authors: M.S.B. 0000-0003-3722-4166; K.G. 0000-0002-1392-8276

Cite this article as: Berkman, M. S., \& Gulec, K. (2021). Pseudo ternary phase diagrams: A practical approach for the area and centroid calculation of stable microemulsion regions. Istanbul Journal of Pharmacy, 51 (1), 42-49.

\begin{abstract}
Background and Aims: This study aims to develop and evaluate a practical approach for the complicated area and centroid calculation of stable microemulsion regions. Pseudo ternary phase diagrams are used to determine the region of microemulsion existence. The effect of various surfactant/co-surfactant weight ratios on the extent of a stable microemulsion area can easily be observed with these diagrams. Furthermore, the optimum formulations are selected using the centroid of the microemulsion region.

Methods: Microemulsion formulations were prepared by changing the weight ratios of the components. The titration method was used at a constant temperature. A pseudo ternary phase diagram was constructed using the spots of the stable microemulsion formulations.

Results: The area and centroid of the stable microemulsion region were calculated by using the formulas manually and the macro edited for Microsoft ${ }^{\circledR}$ Excel. Both results were the same. The macro was user-friendly, easy to use, and worked well in Microsoft ${ }^{\circledR}$ Excel.

Conclusion: Definitions, formulas, algorithms, and calculations used in this research will be constructive for everyone interested in this field and can be modified very easily in every different case.
\end{abstract}

Keywords: Microemulsion, pseudo ternary phase diagram, coordinate geometry, algorithm, Microsoft ${ }^{\circledR}$ Excel macro

\section{INTRODUCTION}

The theoretical existence of microscopic emulsion-like structures in transparent mixtures of water, cationic soap, oil, and alcohol was first put forward by Hoar and Schulman in 1943. These structures were defined as "soluble oil" and "oleopathic hydro-micelle" due to a lack of terminology (Hoar \& Schulman, 1943). In 1959, the term 'microemulsion' was used for the first time by Shulman et al. after electron microscopy visualization of an oil phase stained and solidified system (Schulman, Stoeckenius, \& Prince, 1959; Kreilgaard, 2002). Since then, microemulsions have been found in various applications such as the paint, cosmetic, agriculture, and beverage industries (Spernath \& Aserin, 2006). Microemulsions have also proven themselves as useful drug vehicles for transdermal, dermal, topical, oral, nasal, ocular, and parenteral routes of administration (Fanun, Papadimitriou, \& Xenakis, 2011) as they can confer higher water solubility and bioavailability of drugs (Hathout \& Woodman, 2012).

Microemulsions are thermodynamically stable, isotropic, and transparent dispersions, consisting of an oil and water phase, stabilized by an interfacial surfactant molecular film, usually in conjunction with a cosurfactant (Zhang, Cui, Zhu, Feng, \& Zheng, 
2010). Even there are many more formulation components; it is more convenient to assume the microemulsions as oil/surfactant/water ternary systems (Burguera \& Burguera, 2012). Development of microemulsion-based formulations requires techniques that force the researcher to analyze the microstructure and formation conditions (including analysis of ternary phase diagrams, particle size, and hydration effects, etc.) (Hickey, Hagan, Kudryashov, \& Buckin, 2010). Constructing ternary phase diagrams is a time-consuming process; nevertheless, it is the most important and essential step in the preparation of microemulsion formulation. These diagrams are used to find the region of the microemulsion existence and study the effect of different surfactant/co-surfactant weight ratios on the extent of a stable microemulsion area (Sahoo, Pani, \& Sahoo, 2014). One of the simplest and preferred methods for the ternary phase diagram is to organize and plot the experimental data as oil/water/surfactant percentages. After this step, the optimum formulation is selected using the centroid of the largest microemulsion region. Various Microsoft ${ }^{\circledR}$ Excel templates for the area calculation can be found on the web, but their reliability is suspicious, or the percentage values of the components can be transferred to graph paper to achieve the diagram. However, it is not possible to get the exact results by using this second method because to draw the graph, you need to round a few numbers.

\section{Basic concepts}

Ternary phase diagrams used in microemulsion systems are called 'pseudo ternary phase diagrams' (Schmidts et al., 2009). These are equilateral triangles, and the corners typically represent a binary mixture of two components, such as surfactant/ cosurfactant, water/drug, or oil/drug if the formulation components are more than three (Lawrence \& Rees, 2000). However, generally, each side of the triangle corresponds to one component (oil, surfactant, or water) and is divided into 100 equal parts. Thus, to plot a ternary blend, the mass fraction of the components should be calculated as a percentage by weight (Das et al., 2020).

Two alternative presentations can be used on a ternary phase diagram to identify the component fractions of microemulsion systems. Every spot on the diagram represents a different composition of ternary components, and the mass fractions are read off through the sides of the triangle. The grey arrows show the correct directions to the corresponding component of spot A (40\% oil, 20\% surfactant, and $40 \%$ water) in Figure 1a. If a straight line is parallel to the base of the diagram, it is located opposite to the apex of the triangle. There is only one component at each corner, while the other two do not exist. Parallel lines like B and C are opposite to the apex, where the oil fraction is $100 \%$. Although these lines cut both the oil and surfactant sides, the oil side is the one to be considered. Thus, parallel lines B and C indicate $90 \%$ and $70 \%$ of oil fraction respectively, not the surfactant fraction. The sum of surfactant and water percentage is always 10\% on any point of line B because the sum of the three is always 100\%. The main mistake in this stage is the incorrect drawn parallel lines or not to form $120^{\circ}$ angles between the lines. The mass fractions of the components can also be determined by drawing perpen- dicular lines, as shown in Figure 1b. The shortest distance from any interior spot to each of the three sides is always achieved by a perpendicular line. Viviani's theorem states that 'the sum of these distances equals to the length of altitude in equilateral triangles' (Abboud, 2010).
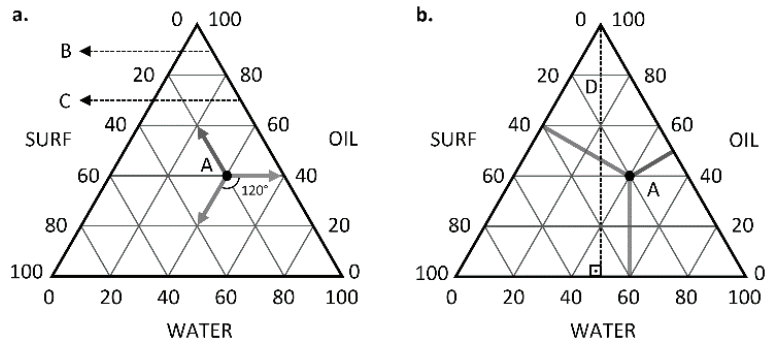

Figure 1. Reading values on a ternary phase diagram.

a. component fractions by parallel lines; b. component fractions by perpendicular lines

Furthermore, the black dashed line D is the altitude and represents $100 \%$ of the oil component. Thus, the distances represented by the grey lines are once again the exact fractions of spot A (40\% oil, $20 \%$ surfactant, and $40 \%$ water) and can easily be achieved.

\section{Coordinate geometry}

The percentages of the three components are useless for the area and centroid calculation unless they are represented in coordinate geometry. The problem starts at that point, as it is a hard step to transpose the spots into coordinates. In fact, the solution is very simple with the help of basic trigonometry knowledge. Although the oil, surfactant, and water percentages for spot A are known, what is the exact coordinate of this formulation on the $x$ and $y$ axes? Figure 2 provides a closer look at the situation and represents the phase diagram on coordinate geometry.

There is no need to rescale the triangle if 100 units of length are used on both axes. Thus, the base of the triangle is 100 units. A perpendicular line to the $x$-axis gives the $x$-coordinate as 60 units. Luckily, these phase diagrams are equilateral triangles with an interior angle of $60^{\circ}$ in each corner. The cosine function can be used to achieve the $y$-coordinate because the cosine of an angle is the ratio of the length of the adjacent side (y) to the length of the hypotenuse, where it represents the oil percentage in Figure 2.

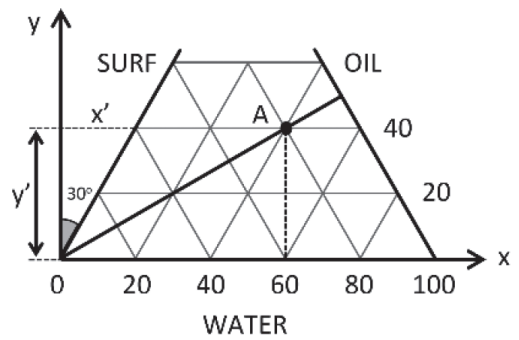

Figure 2. Pseudo ternary phase diagram on coordinate geometry. 
$y^{\prime}=$ hypotenuse $\mathrm{x} \cos \left(30^{\circ}\right)=40 \times \cos \left(30^{\circ}\right)=34.6410$

Consequently, the exact coordinate of the spot is $A(60.0000,34.6410)$. This approach is practical and works theoretically, but the $x$-coordinate is not always on the intersection of small triangles nor so easily readable. Actually, the fractions of the components obtained from experimental results are between the grey guidelines, as shown in Table 1.

\section{Table 1. Component fractions obtained from the experimental titration results.}

\begin{tabular}{|lccc|}
\hline & \multicolumn{3}{c}{ Components (\%) } \\
\cline { 2 - 4 } Spots & Oil & Surfactant & Water \\
\hline A & 18 & 68 & 14 \\
B & 26 & 57 & 17 \\
C & 42 & 46 & 12 \\
D & 53 & 36 & 11 \\
E & 71 & 22 & 7 \\
\hline
\end{tabular}

If all the formulation data are displayed on a ternary phase diagram, the location of the spots in the triangle can be observed clearly. This time the spots are not on the guidelines as shown in Figure 3.

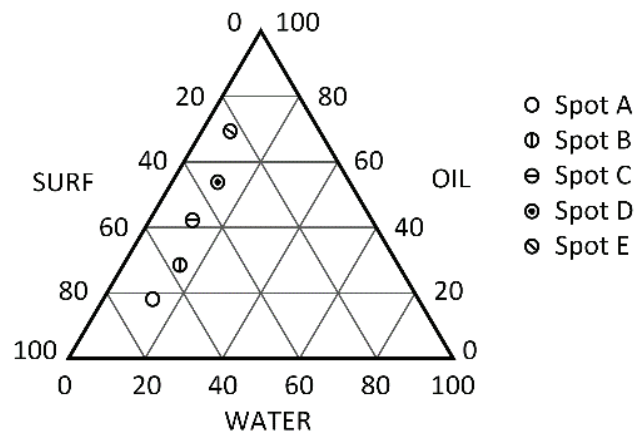

Figure 3. Plotting the spots on a pseudo ternary phase diagram.

After plotting spots A to E on the diagram, the same method with trigonometric functions can be used to define the coordinates. The first step to be done is to transpose the spots into coordinates. If the coordinates are carried out for spot $\mathrm{A}$, then the formula can be used for the other spots as well. Therefore, a closer look at spot A will be useful, as in Figure 4.

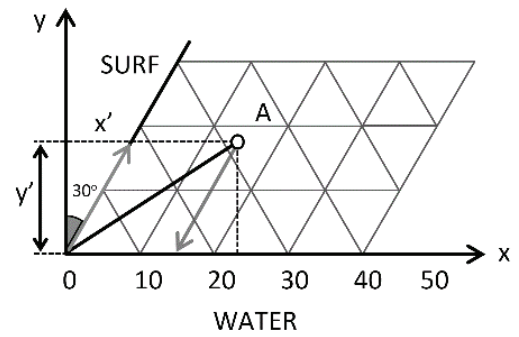

Figure 4. A closer look at a section of the pseudo ternary phase diagram.
Neither the $x$ nor the $y$-coordinates can be read directly in Figure 4 . The only known data are oil, surfactant, and water percentages, which are 18,68 , and 14 , respectively. First, let us use the cosine function to achieve the $y$-coordinate $(y)$. As previously mentioned, note that the length of the hypotenuse (grey arrows) also represents the oil percentage in Figure 4.

$y^{\prime}=$ hypotenuse $\mathrm{x} \cos \left(30^{\circ}\right)=18 \times \cos \left(30^{\circ}\right)=15.5885$

$y_{A}=15.5885$

Before finding the exact $x$-coordinate, the length of $x^{\prime}$ has to be calculated by using a similar equation to $y^{\prime}$;

$x^{\prime}=$ hypotenuse $\times \sin \left(30^{\circ}\right)=18 \times \sin \left(30^{\circ}\right)=9.0000$

The downward grey arrow in Figure 4 and also Figure $1 \mathrm{a}$ is used to detect the water percentage, and this line is parallel to the hypotenuse as well. Thus, the sum of water percentage and $x^{\prime}$ is equal to the $x$-coordinate $\left(x_{A}\right)$ of the spot.

$x_{A}=$ water percentage $+x^{\prime}=14+9.0000=23.0000$

The exact coordinate of the spot is $A(23.0000,15.5885)$. Briefly, two general formulas can be used in every spot to determine the $x$ and $y$-coordinates. These are

$y_{i}=($ oil percentage $) \times \cos \left(30^{\circ}\right)$

$x_{i}=($ water percentage $)+\left\{(\right.$ oil percentage $\left.) \times \sin \left(30^{\circ}\right)\right\}$

If Microsoft ${ }^{\circledR}$ Excel is preferred instead of using a calculator, the following functions can be applied to the cells;

$=\left(\operatorname{COS}(\operatorname{RADIANS}(30))^{*}(\mathrm{OIL}\right.$ CELL $\left.)\right)$ for $y_{i}$

$=\left(\operatorname{SIN}(\text { RADIANS }(30))^{*}(\right.$ OIL CELL $\left.)\right)+($ WATER CELL $)$ for $x_{i}$

All the coordinates of spot A to E are given in Table 2, with the corresponding percentage of components.

\section{Table 2. The coordinates of spots and the percentage of the components.}

\begin{tabular}{|lcc|}
\hline \multirow{2}{*}{ Spots } & \multicolumn{2}{c|}{ Coordinates } \\
\cline { 2 - 3 } & \multicolumn{1}{c|}{$\boldsymbol{x}_{\boldsymbol{i}}$} & \multicolumn{1}{c|}{$\boldsymbol{y}_{\boldsymbol{i}}$} \\
\hline A & 23.0000 & 15.5885 \\
B & 30.0000 & 22.5167 \\
C & 33.0000 & 36.3731 \\
D & 37.5000 & 45.8993 \\
E & 42.5000 & 61.4878 \\
\hline
\end{tabular}

\section{Creating a closed region}

In order to select the optimum formulation and investigate the area and centroid, a closed region (Figure 5) in the ternary phase diagram should be defined. Thus, two straight lines are drawn from the corner to the opposite side, covering all the spots A to E (Yang, Kim, \& Kim, 2002). The component on 


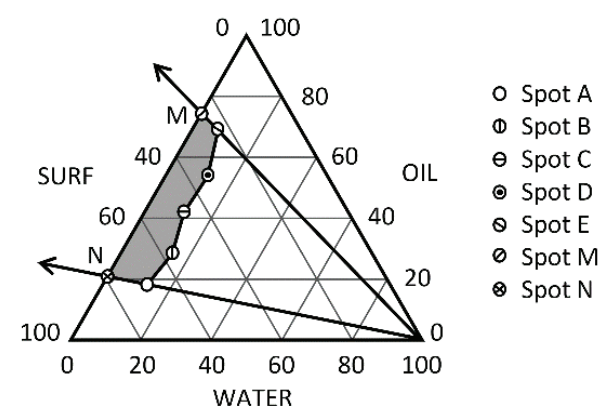

Figure 5. Stable microemulsion region on the pseudo ternary phase diagram.

the selected corner is called the formulation variable. If the microemulsion formulation is constructed by a water titration method, then the formulation variable is water (Liu et al., 2013).

When a closed region is formed with the boundary lines, two more spots must be considered. After these steps, there will be seven coordinated spots for the area calculation. Although the same methods are used for the coordinate calculation, the process is slightly more complicated. If the coordinates are carried out for spot $\mathrm{N}$, then the formula can be used for spot $M$ as well. Therefore, once again, a closer look at spot $\mathrm{N}$ will be useful, as in Figure 6a. If two straight lines are non-parallel on a two-dimensional space, they intersect at only one spot, which can be described by a single set of $(x, y)$ coordinates (Figure 6b).

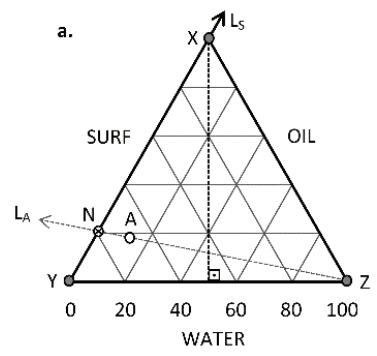

b.

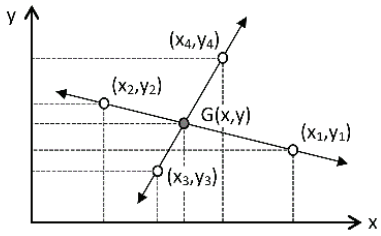

Figure 6. Position of spot $\mathrm{N}$ on pseudo ternary phase diagram. a. intersection of line $L_{A}$ and $L_{S}$ on coordinate geometry; b. the intersection of two straight lines.

Spot $N(x, y)$ is the intersection of line $A\left(L_{A}\right)$ and the surfactant side of the triangle $\left(L_{S}\right)$. Thus, to assign $N(x, y)$, each line has to be defined by two distinct spots with known coordinates, which may be as;

$$
\begin{aligned}
& Z\left(x_{1}, y_{1}\right)=Z(100.0000,0.0000) \text { and } \\
& A\left(x_{2}, y_{2}\right)=A(23.0000,15.5885) \text { for } L_{A} \\
& Y\left(x_{3}, y_{3}\right)=Y(0.0000,0.0000) \text { and } \\
& X\left(x_{4}, y_{4}\right)=X(50.0000,86.6025) \text { for } L_{S}
\end{aligned}
$$

The intersection coordinate $G(x, y)$ of line $L_{A}$ and $L_{S}$ can be defined as;

$$
\begin{aligned}
& x_{G}=\frac{\left(x_{1} y_{2}-y_{1} x_{2}\right)\left(x_{3}-x_{4}\right)-\left(x_{1}-x_{2}\right)\left(x_{3} y_{4}-y_{3} x_{4}\right)}{\left(x_{1}-x_{2}\right)\left(y_{3}-y_{4}\right)-\left(y_{1}-y_{2}\right)\left(x_{3}-x_{4}\right)} \\
& y_{G}=\frac{\left(x_{1} y_{2}-y_{1} x_{2}\right)\left(y_{3}-y_{4}\right)-\left(y_{1}-y_{2}\right)\left(x_{3} y_{4}-y_{3} x_{4}\right)}{\left(x_{1}-x_{2}\right)\left(y_{3}-y_{4}\right)-\left(y_{1}-y_{2}\right)\left(x_{3}-x_{4}\right)}
\end{aligned}
$$

After a series of laborious steps, the calculated intersection coordinate is;

$$
N(x, y)=N(10.4651,18.1261)
$$

The usage of this formula is quite hard unless the data are transferred to Microsoft ${ }^{\circledR}$ Excel or any other calculus software. But there is quite a simple second way by using the previously mentioned equations and similarity.

Figure 7 represents two similar triangles with common sides. Thus, a simple proportion between them can be set;

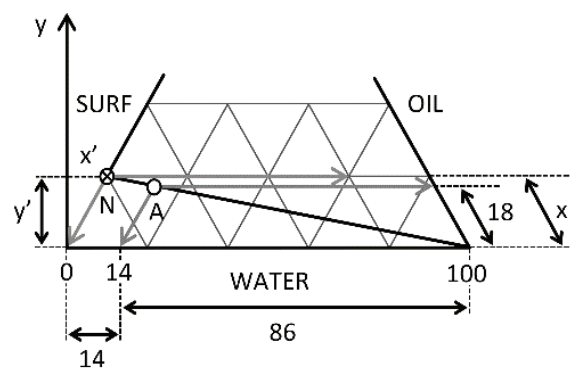

Figure 7. Position of spot $\mathrm{N}$ on pseudo ternary phase diagram by similarity.

$\frac{86}{100}=\frac{18}{x} \quad x=20.9302$

This $x$ value is the oil fraction of spot N, where the water fraction is zero. The exact coordinates of spot $\mathrm{N}$ can be achieved by using the previous equations, respectively.

$y_{N}=20.9302 \times \cos \left(30^{\circ}\right)=18.1261$
$x_{N}=20.9302 \times \sin \left(30^{\circ}\right)=10.4651$

The coordinates of the seventh spot, spot M, can be achieved as well with the same steps;

$$
\begin{aligned}
& \frac{93}{100}=\frac{71}{x} \quad x=76.3441 \\
& y_{M}=76.3441 \times \cos \left(30^{\circ}\right)=66.1159 \\
& x_{M}=76.3441 \times \sin \left(30^{\circ}\right)=38.1720
\end{aligned}
$$

\section{Area and centroid calculation}

Calculating the area of a polygon is complicated. It can be verified by partitioning the polygon into smaller shapes, such as triangles or other quadrilaterals. Individual areas are calculated 
and added together to find the entire polygon area. But the more numbers you deal with, the higher the chance of making mistakes. On the other hand, Gauss's area formula (Shoelace formula) and its mathematical algorithm offer a much easier way to determine the area (Lee, \& Lim, 2017). Consider a nonself-intersecting closed polygon with $n$ sides (Figure 8 ) and list the coordinates of each vertex clockwise or counterclockwise as you wish.

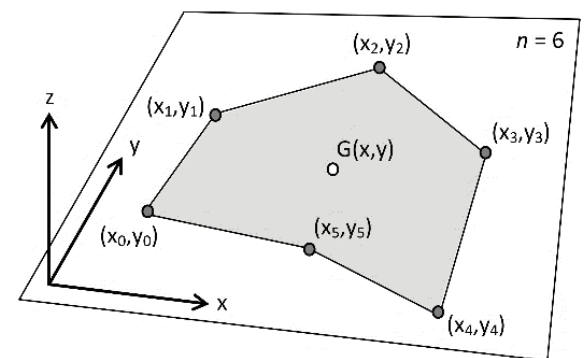

Figure 8. The coordinates of a closed polygon.

Notice that the polygon is closed. Thus, the last vertex $\left(x_{n+1}, y_{n+1}\right)$ is the starting coordinate. The formula used is similar to a determinant function and can be expressed as below.

$$
A=\frac{1}{2}\left|\begin{array}{lllll}
x_{1} & x_{2} & \cdots & x_{n} & x_{1} \\
y_{1} & y_{2} & \cdots & y_{n} & y_{1}
\end{array}\right|
$$

It is not literally a true determinant because the matrix is not square but can be evaluated like two by two determinants as well. Starting with $x_{1}$, multiply the diagonal pairs and add them. Essentially, you get the sum of $x_{1} y_{2}+x_{2} y_{3}+\ldots+x_{n} y_{1}$. Starting with $y_{1}$, do the same calculations and get the sum of $y_{1} x_{2}+y_{2} x_{3}+\ldots+y_{n} x_{1}$. The value of the determinant is the difference between these two terms, and if the result is negative, then use the absolute value. Multiply it by half to get the area. This formula can be used for any closed polygons and represented by the expression below.

$A=\frac{1}{2}\left|\sum_{i=1}^{n-1} x_{i} y_{i+1}+x_{n} y_{1}-\sum_{i=1}^{n-1} x_{i+1} y_{i}+x_{1} y_{n}\right|$

$A=\frac{1}{2}\left|x_{1} y_{2}+x_{2} y_{3}+\cdots+x_{n} y_{1}-x_{2} y_{1}-\cdots-x_{1} y_{n}\right|$

This formula can be simplified as;

$$
A=\frac{1}{2}\left|\sum_{i=1}^{n}\left(x_{i} y_{i+1}-x_{i+1} y_{i}\right)\right|
$$

Alternatively, the Trapezoid Rule can also be used to determine the area of polygons (Smith, Goodchild, \& Longley, 2007). The area between the $x$-axis and the two vertical lines at $x_{1}$ and $x_{2}$ is simply the width between the two lines $\left(x_{2}-x_{1}\right)$ times the average height of the two lines $\left(y_{1}+y_{2}\right) / 2$. If these operations are performed for all coordinates and the areas are added with positive and negative values, the total area of the polygon is obtained. In this case, the formula can be rearranged as;
$A=\frac{1}{2}\left|\sum_{i=1}^{n}\left(x_{i+1}-x_{i}\right)\left(y_{i}+y_{i+1}\right)\right|$

According to Green's Theorem (Green \& Thomson, 1828; Soerjadi, 1968), if a curve $C$ forms the boundary of a region $D$ and $F_{n}$ 's are the functions of $(x, y)$ defined on an open region containing $D$ with continuous partial derivatives there, then it states;

$\oint_{C} F_{1}(x, y) d x+F_{2}(x, y) d y=\iint_{D}\left(\frac{\partial F_{2}}{\partial x}-\frac{\partial F_{1}}{\partial y}\right) d x d y$

This theorem can only be used for the area calculation of a polygon in the following case;

$\frac{\partial F_{2}}{\partial x}-\frac{\partial F_{1}}{\partial y}=1$

Choosing $F_{1}(x, y)=0$ and $F_{2}(x, y)=x$ meets this requirement. Then, the area of the region can also be calculated by evaluating any of the following line integrals.

$A=\oint_{C} x d y=-\oint_{C} y d x=\frac{1}{2} \oint_{C}(x d y-y d x)$

Line integrals over the curves are additive over length, so;

$A=\oint_{C} x d y=\int_{C_{1}} x d y+\int_{C_{2}} x d y+\cdots+\int_{C_{n}} x d y$

After the segment parametrisation from $\left(x_{i}, y_{i}\right)$ to $\left(x_{i+1}, y_{i+1}\right)$ and the integral substitution, the sum of all $C_{i}$ gives the total area same as the Trapezoid Rule.

$C_{i}: \vec{r}=\left(\left(x_{i+1}-x_{i}\right) t+x_{i},\left(y_{i+1}-y_{i}\right) t+y_{i}\right), \quad 0 \leq t \leq 1$

$$
\begin{aligned}
\int_{C_{i}} x d y & =\left(y_{i+1}-y_{i}\right) \int_{0}^{1}\left(\left(x_{i+1}-x_{i}\right) t+x_{i}\right) d t \\
& =\frac{\left(x_{i}+x_{i+1}\right)\left(y_{i+1}-y_{i}\right)}{2}
\end{aligned}
$$

$A=\frac{1}{2}\left|\sum_{i=1}^{n}\left(x_{i}+x_{i+1}\right)\left(y_{i+1}-y_{i}\right)\right|$

After the area is calculated, one more parameter must be determined. The centroid of a non-self-intersecting closed polygon defined by $n$ vertices $\left(x_{1}, y_{1}\right),\left(x_{2}, y_{2}\right), \ldots,\left(x_{n}, y_{n}\right)$ is the point $\left(G_{x}, G_{y}\right)$, where;

$$
\begin{aligned}
& G_{x}=\frac{1}{6 A} \sum_{i=0}^{n-1}\left(x_{i}+x_{i+1}\right)\left(x_{i} y_{i+1}-x_{i+1} y_{i}\right) \\
& G_{y}=\frac{1}{6 A} \sum_{i=0}^{n-1}\left(y_{i}+y_{i+1}\right)\left(x_{i} y_{i+1}-x_{i+1} y_{i}\right)
\end{aligned}
$$


Although these formulas are beneficial on their own, it is important to consider that this approach involves a lot of tedious arithmetic calculations. Thus, its main benefit arises when applied in a computer program as an algorithm.

\section{Model optimization and algorithm}

Microsoft ${ }^{\circledR}$ Excel is one of the most used software programs of all time. Millions of people around the world use this software to enter all sorts of data and perform financial, mathematical, or statistical calculations. It allows us to organize, format, and calculate data with formulas using a spreadsheet system. In addition to its standard calculation features, Excel also offers the ability to access data from external sources via Microsoft's Dynamic Data Exchange (DDE), programming support via Microsoft's Visual Basic for Applications (VBA), and extensive graphing and charting capabilities. Due to all these features, it can be said that this software is a very advanced calculator.

VBA is one of the most powerful features of Microsoft ${ }^{\circledR}$ Excel. It enables us to write functions and subroutines. A function returns a value to a cell in the same way as a worksheet function while a subroutine performs a process. Briefly, subroutines and functions are known as macros, and we need to use these userdefined functions (UDF) beside the built-in ones. If Microsoft ${ }^{\circledR}$ Excel has no built-in function, UDFs may be more convenient when we repeatedly need to perform a specific type of calculation (Liengme, 2016).

In order to work with the VBA editor, we shall need to have the Developer tab on the ribbon. If it is not present, right-click any of the tabs on the ribbon and select Customize Ribbon, and in the right-hand panel, enter a checkmark in the Developer box. Click the OK button to close the dialog.

The visual basic editor allows us to calculate the area and centroid of the stable microemulsion regions by using the formulas mentioned above. But first, we need to create an algorithm to make these operations simpler and write it into the relevant box. Open Microsoft ${ }^{\circledR}$ Excel, copy your data to the spreadsheet, and then use the command Developer / Code / Visual Basic (or the shortcut Alt+F1) to open the VBE. Use the command Insert / Module and add a module to the project tree. Click the module1 section and type or copy/ paste the algorithm below. Do not forget to save your work as Macro-Enabled Workbook.

$$
\begin{aligned}
& \text { Function AREA(Coordinate) } \\
& \text { mylast }=\text { Coordinate.Count } / 2 \\
& \begin{aligned}
& \text { Area } 1=0 \\
& \text { For } I=1 \text { To mylast } \\
& x i=\text { Coordinate }(l, 1) \\
& y i=\text { Coordinate }(l, 2) \\
&|f|=\text { mylast Then } \\
& x k=\text { Coordinate }(1,1) \\
& y k=\text { Coordinate }(1,2) \\
& \text { Else }
\end{aligned}
\end{aligned}
$$

$$
x k=\text { Coordinate }(l+1,1)
$$

$y k=$ Coordinate $(l+1,2)$

End If

$$
\begin{aligned}
& \text { Formula }=\left(x{ }^{*} y k-x k^{*} y i\right) \\
& \text { Area1 }=\text { Area1 + Formula }
\end{aligned}
$$

Next I

$$
A R E A=A b s(\text { Area } 1 / 2)
$$

End Function

\section{Function GX(Coordinate)}

mylast $=$ Coordinate.Count $/ 2$

Area $1=0$

$$
c x=0
$$

$$
\begin{aligned}
& \text { For } I=1 \text { To mylast } \\
& x i=\text { Coordinate }(I, 1) \\
& y i=\text { Coordinate }(I, 2) \\
& |f|=\text { mylast Then } \\
& x k=\text { Coordinate }(1,1) \\
& y k=\text { Coordinate }(1,2) \\
& \text { Else } \\
& x k=\text { Coordinate }(I+1,1) \\
& y k=\text { Coordinate }(I+1,2) \\
& \text { End If } \\
& \text { Formula }=(x i * y k-x k * y i) \\
& \text { Areal }=\text { Areal }+ \text { Formula } \\
& \text { Cx }=C x+(x i+x k) * \text { Formula }
\end{aligned}
$$

Next 1

$$
G X=\operatorname{Abs}(C X /(3 * \text { Area } 1))
$$

\section{End Function}

\section{Function GY(Coordinate)}

mylast $=$ Coordinate.Count $/ 2$

Area $1=0$

$\mathrm{Cy}=0$

$$
\begin{aligned}
& \text { For } I=1 \text { To mylast } \\
& x i=\text { Coordinate }(I, 1) \\
& y i=\text { Coordinate }(I, 2) \\
& \mid f I=\text { mylast Then } \\
& x k=\text { Coordinate }(1,1) \\
& y k=\text { Coordinate }(1,2) \\
& \text { Else } \\
& x k=\text { Coordinate }(I+1,1) \\
& y k=\text { Coordinate }(I+1,2) \\
& \text { End If } \\
& \text { Formula }=(x i * y k-x k * y i) \\
& \text { Areal }=\text { Area } 1+\text { Formula } \\
& \text { Cy }=\text { Cy }+(y i+y k) * \text { Formula } \\
& \text { Next I } \\
& \text { GY }=\text { Abs }(C y /(3 * \text { Area } 1))
\end{aligned}
$$

End Function 
If there is no problem, the macro is ready to use. Return to the Microsoft ${ }^{\circledR}$ Excel main page (you can click the Excel main file in the Windows taskbar or the Excel icon on the VBE toolbar) and just type the new function code as any of the built-in functions. Your new assigned functions are;

\author{
$=\operatorname{AREA}\left(x_{i}\right.$ coordinates, $y_{i}$ coordinates $)$ \\ $=\mathrm{GX}\left(x_{i}\right.$ coordinates, $y_{i}$ coordinates $)$ \\ $=\mathrm{GY}\left(x_{i}\right.$ coordinates, $y_{i}$ coordinates $)$
}

\section{MATERIALS AND METHOD}

This section provides details of the materials and methodology used to get the data needed for the calculations.

\section{Materials}

The following materials were used for the preparation of the microemulsion formulations. For the oil phase, isopropyl myristate (IPM), was provided by Merck Schuchardt OHG (Germany). The surfactants polyoxyethylene sorbitan monooleate (Tween ${ }^{\circledR} 80$ ) and sorbitan monolaurate (Span ${ }^{\circledR} 20$ ) were from Sigma-Aldrich (Germany). Ultrapure water (Millipore Milli-Q, USA) was used throughout the experiments as the hydrophilic phase. All chemicals were of pharmaceutical grade and used as received without any further purification.

\section{Pseudo ternary phase diagram}

A pseudo ternary phase diagram was constructed to find the area of microemulsion existence. Briefly, Tween ${ }^{\circledR} 80$ and Span $^{\circledR} 20$ were blended in a 1:1 weight ratio and added into the oil phase. Ten grams of IPM and surfactant mixtures were prepared with various weight ratios from 1.0:9.0 to 9.0:1.0 in glass beakers. Ultrapure water was added dropwise to each oily mixture while stirring on a mechanical stirrer (200 rpm) at room temperature $\left(25 \pm 1^{\circ} \mathrm{C}\right)$. The titration was continued until the transparent and homogeneous dispersion turned turbid, which defined the transition point from microemulsion to coarse emulsion. The amount of water required to turn the mixture slightly turbid was then recorded. The samples were allowed to equilibrate at $25 \pm 1^{\circ} \mathrm{C}$ for at least $24 \mathrm{~h}$, and the transparent samples were considered as monophasic spots in the phase diagram (Figure 9).

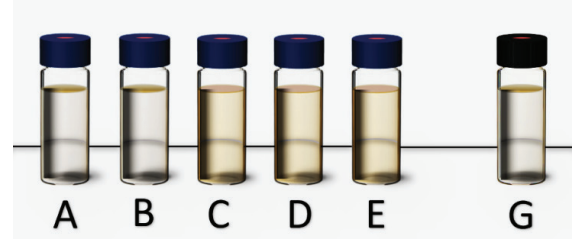

Figure 9. Transparent microemulsion samples (A-E) and the final formulation prepared from the $G$ spot.

The mass fractions (oil, surfactants, and water) of each spot were calculated and plotted in the pseudo ternary phase diagram to find the stable microemulsion region. The percentages of the components and the coordinates of the spots are presented in Table 1, Table 2, and Figure 5 as well.

\section{RESULTS AND DISCUSSION}

The area and centroid of the stable microemulsion region were calculated using the formulas manually and the macro edited for Microsoft ${ }^{\circledR}$ Excel. Both results were the same.

The macro was super easy to use and available in the $f x$ function menu too. We have worked very hard to make this algorithm user-friendly. The main problem in the development process was the desire to achieve results without the repetition of the last vertex because, as previously mentioned, $\left(x_{n+1}, y_{n+1}\right)$ was the starting coordinate. In detail, the problem was solved, and unlike the formulas, you do not need to enter the first vertex again at the end, and you can go in either direction around the polygon. The internal programming of the calculator takes care of it all for you. A sample section of Microsoft ${ }^{\circledR}$ Excel interface while using the algorithm and the functions is present in Figure 10.

\begin{tabular}{|c|c|c|c|c|c|}
\hline \multicolumn{2}{|r|}{$\vdots$} & $\checkmark$ & \multicolumn{2}{|c|}{$=A R E A(C 2: D 8)$} & \\
\hline$\triangle \mathrm{B}$ & C & D & E & $\mathrm{F}$ & G \\
\hline 1 & $x i$ & $y i$ & & & \\
\hline 2 & 23.0000 & 15.5885 & & Area & 556.5882 \\
\hline 3 & 30.0000 & 22.5167 & & Gx & 27.6889 \\
\hline 4 & 33.0000 & 36.3731 & & $=\mathrm{Gy}$ & 36.9646 \\
\hline 5 & 37.5000 & 45.8993 & & $f\left(f_{x} \mathrm{GY}\right.$ & \\
\hline 6 & 42.5000 & 61.4878 & & $\begin{array}{ll}f x & G X \\
\end{array}$ & \\
\hline 7 & 38.1720 & 66.1159 & & \begin{tabular}{l|l}
$f_{x}$ & AREA
\end{tabular} & \\
\hline 8 & 10.4651 & 18.1261 & & & \\
\hline 9 & & & & & \\
\hline
\end{tabular}

Figure 10. The area and centroid results in Microsoft ${ }^{\circledR}$ Excel.

The area of the stable microemulsion region was 556.5886 unit squared, and the centroid was on the $G(27.6889,36.9646)$ spot, as seen in Figure 9 and 10. After this step, we needed a final calculation to find the percentage values of the components. The results were easily achieved by a reverse calculation specified at the beginning of the manuscript.

$$
\begin{aligned}
\text { oil percentage } & =G_{y} / \cos \left(30^{\circ}\right) \\
& =36.9646 / \cos \left(30^{\circ}\right) \\
& =42.6830 \approx 43 \%
\end{aligned}
$$

water percentage $=G_{x}-\left\{(\right.$ oil percentage $\left.) \mathrm{x} \sin \left(30^{\circ}\right)\right\}$

$$
\begin{aligned}
& =27.6889-\left\{42.6830 \times \sin \left(30^{\circ}\right)\right\} \\
& =6.3474 \approx 6 \%
\end{aligned}
$$

surfactants percentage $=100-(42.6830+6.3474)$

$$
=50.9696 \approx 51 \%
$$

Remember that the surfactants were blended in a 1:1 weight ratio; therefore, the percentages of Tween ${ }^{\circledR} 80$ and Span ${ }^{\circledR} 20$ are both $25.5 \%$.

In fact, the coordinate and percentage calculations could also be included in the algorithm. However, the locations of the components are interchangeable, and it was preferred to keep 
it simple. This version of the algorithm could be used much more comfortably as a source code for Microsoft ${ }^{\circledR}$ Excel and all other platforms.

\section{CONCLUSION}

Microemulsion formulations were prepared by changing the weight ratios of the components. The titration method was used at a constant temperature. The pseudo ternary phase diagram was constructed using the spots of the stable microemulsion formulations. The region of microemulsion formation was assigned, then the area and centroid of the region were calculated. Formulas and the algorithm were used for all the calculations. A final formulation was prepared according to the percentages on the centroid and found to be stable as expected.

As a result, the macro was user-friendly, easy-to-use, and worked well in Microsoft ${ }^{\circledR}$ Excel. Definitions, formulas, and calculations used in this study will be very helpful for everyone interested in this field and can be modified very easily in every different case.

Acknowledgment: The authors thank the research assistant Ismail Terzi (Eskişehir Technical University, Department of Computer Engineering) for his valuable contributions to VBA and macro editing.

Peer-review: Externally peer-reviewed.

Author contributions: Conception/Design of Study- M.S.B., K.G.; Data Acquisition- M.S.B.; Data Analysis/Interpretation- M.S.B., K.G.; Drafting Manuscript-M.S.B., K.G.; Critical Revision of Manuscript- M.S.B.; Final Approval and Accountability- M.S.B., K.G.

Conflict of Interest: The authors have no conflict of interest to declare.

Financial Disclosure: Authors declared no financial support.

\section{REFERENCES}

- Abboud, E. (2010). Viviani's theorem and its extension. The College Mathematics Journal, 41(3), 203-211.https://doi. org/10.4169/074683410X488683

- Burguera, J. L., \& Burguera, M. (2012). Analytical applications of emulsions and microemulsions. Talanta, 96, 11-20.https://doi. org/10.1016/j.talanta.2012.01.030

- Das, S., Lee, S. H., Chia, V. D., Chow, P. S., Macbeath, C., Liu, Y., \& Shliout, G. (2020). Development of microemulsion based topical ivermectin formulations: pre-formulation and formulation studies. Colloids and Surfaces B: Biointerfaces, 189, 1-8.https://doi. org/10.1016/j.colsurfb.2020.110823

- Fanun, M., Papadimitriou, V., \& Xenakis, A. (2011). Characterization of cephalexin loaded nonionic microemulsions. Journal of Colloid and Interface Science, 362(1), 115-121.https://doi.org/10.1016/j. jcis.2011.05.042

- Green, G., \& Thomson, W. (1828). An essay on the application of mathematical analysis to the theories of electricity and magnetism. Nottingham: T Wheelhouse.

- Hathout, R. M., \&Woodman, J. (2012). Applications of NMR in the characterization of pharmaceutical microemulsions. Journal of Controlled Release, 161(1), 62-72. https://doi.org/10.1016/j.jconrel.2012.04.032
Hickey, S., Hagan, S. A., Kudryashov, E., \& Buckin, V. (2010). Analysis of phase diagram and microstructural transitions in an ethyl oleate/water/Tween 80/Span 20 microemulsion system using high-resolution ultrasonic spectroscopy. International Journal of Pharmaceutics, 388(1-2), 213-222.https://doi.org/10.1016/j. ijpharm.2009.12.003

- Hoar, T. P., \& Schulman, J. H. (1943). Transparent water in oil dispersions: oleopathic hydromicelle. Nature, 152, 102-103.https://doi. org/10.1038/152102a0

- Kreilgaard, M. (2002). Influence of microemulsions on cutaneous drug delivery. Advanced Drug Delivery Reviews, 54(1), 77-98. https://doi.org/10.1016/S0169-409X(02)00116-3

- $\quad$ Lawrence, M. J., \& Rees, G. D. (2000). Microemulsion-based media as novel drug delivery systems. Advanced Drug Delivery Reviews, 45(1), 89-121.https://doi.org/10.1016/S0169-409X(00)00103-4

- Lee, Y., \& Lim, W. (2017). Shoelace formula: connecting the area of a polygon with vector cross product. The Mathematics Teacher, 110(8), 631-636.https://doi.org/10.5951/mathteacher.110.8.0631

- Liengme, B. V. (2016). VBA user-defined functions. In B. V. Liengme (Ed.), A guide to Microsoft Excel 2013 for scientists and engineers (pp. 181-206). San Diego: Academic Press.https://doi.org/10.1016/ C2014-0-03421-1

- Liu, D., Kobayashi, T., Russo, S., Li, F., Plevy, S. E., Gambling, T. M., Carson, J. L., \& Mumper, R. J. (2013). In vitro and in vivo evaluation of a water-in-oil microemulsion system for enhanced peptide intestinal delivery. The AAPS Journal, 15(1), 288-298.https://doi. org/10.1208/s12248-012-9441-7

- Sahoo, S., Pani, N. R., \& Sahoo, S. K. (2014). Microemulsion based topical hydrogel of sertaconazole: Formulation, characterization and evaluation. Colloids and Surfaces B: Biointerfaces, 120, 193-199. https://doi.org/10.1016/j.colsurfb.2014.05.022

- Schmidts, T., Nocker, P., Lavi, G., Khulman, J., Czermak, P., \& Runkel, F. (2009). Development of an alternative, time and cost saving method of creating pseudoternary diagrams using the example of a microemulsion. Colloids and Surfaces A: Physicochemical and Engineering Aspects, 340(1-3), 187-192. https://doi.org/10.1016/j. colsurfa.2009.03.029

- Schulman, J. H., Stoeckenius, W., \& Prince, L.M. (1959). Mechanism of formation and structure of microemulsions by electron microscopy. The Journal of Physical Chemistry, 63(10), 1677-1680. https:// doi.org/10.1021/j150580a027

Smith, M. J., Goodchild, M. F., \& Longley, P. A. (2007). Building blocks of spatial analysis. In M. J. Smith, M. F. Goodchild \& P. A. Longley (Eds.), Geospatial analysis: A comprehensive guide to principles, techniques and software tools (pp. 69-71). United Kingdom: Matador.

- Soerjadi, R. (1968). On the computation of the moments of a polygon, with some applications. Heron, 16(5), 43-58.

- Spernath, A., \& Aserin, A. (2006). Microemulsions as carriers for drugs and nutraceuticals. Advances in Colloid and Interface Science, 128-130, 47-64.https://doi.org/10.1016/j.cis.2006.11.016

- Yang, J. H., Kim, Y., \& Kim, K. M. (2002). Preparation and evaluation of aceclofenac microemulsion for transdermal delivery system. Archives of Pharmacal Research, 25(4), 534-540.https://doi. org/10.1007/BF02976614

- $\quad$ Zhang, H., Cui, Y., Zhu, S., Feng, F., \& Zheng, X. (2010). Characterization and antimicrobial activity of a pharmaceutical microemulsion. International Journal of Pharmaceutics, 395(1-2), 154-160. https://doi.org/10.1016/j.jpharm.2010.05.022 